\title{
In situ TEM probing of crystallization form-dependent sodiation behavior in ZnO nanowires for sodium-ion batteries
}

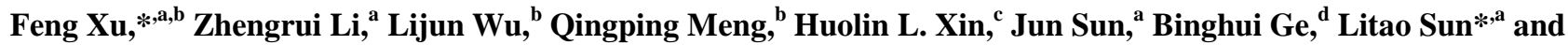 \\ Yimei Zhu*,b
}

${ }^{\text {a } S E U-F E I ~ N a n o-P i c o ~ C e n t e r, ~ K e y ~ L a b o r a t o r y ~ o f ~ M E M S ~ o f ~ t h e ~ M i n i s t r y ~ o f ~ E d u c a t i o n, ~ S o u t h e a s t ~ U n i v e r s i t y, ~ N a n j i n g ~}$ 210096, China

${ }^{\mathrm{b}}$ Condensed Matter Physics \& Materials Science Department, Brookhaven National Laboratory, Upton, NY 11973, USA

${ }^{\mathrm{c} C e n t e r}$ for Functional Nanomaterials, Brookhaven National Laboratory, Upton, NY11973, USA

${ }^{\mathrm{d}}$ Institute of Physics, Chinese Academy of Sciences, Beijing 100190, China

*Corresponding author. E-mail address: fxu@seu.edu.cn (F. Xu), slt@ seu.edu.cn (L. Sun), and zhu@bnl.gov (Y.Zhu).

\begin{abstract}
Development of sodium-ion battery (SIB) electrode materials currently lags behind electrodes in commercial lithium-ion batteries (LIBs). However, in the long term, development of SIB components is a valuable goal. Their similar, but not identical, chemistries require careful identification of the underlying sodiation mechanism in SIBs. Here, we utilize in situ transmission electron microscopy to explore quite different sodiation behaviors even in similar electrode materials through real-time visualization of microstructure and phase evolution. Upon electrochemical sodiation, single-crystalline $\mathrm{ZnO}$ nanowires (sc-ZNWs) are found to undergo a step-by-step electrochemical displacement reaction, forming crystalline $\mathrm{NaZn}_{13}$ nanograins dispersed in a $\mathrm{Na}_{2} \mathrm{O}$ matrix. This process is characterized by a slowly propagating reaction front and the formation of heterogeneous interfaces inside the ZNWs due to non-uniform sodiation amorphization. In contrast, poly-crystalline ZNWs (pc-ZNWs) exhibited an ultrafast sodiation process, which can partly be ascribed to the availability of unobstructed ionic transport pathways among $\mathrm{ZnO}$ nanograins. Thus the reaction front and heterogeneous interfaces disappear. The in situ TEM results, supported by calculation of the ion diffusion coefficient, provide breakthrough insights into the dependence of ion diffusion kinetics on crystallization form. This points toward a goal of optimizing the microstructure of electrode materials in order to develop high performance SIBs.
\end{abstract}

Keywords: Sodium-ion batteries; In situ transmission electron microscopy; ZnO nanowires; Electrochemical sodiation; Microstructure control 


\section{Introduction}

Rechargeable lithium-ion batteries (LIBs) have become a mainstay of the digital age with dominance in portable electronics market. However, for emerging large-scale stationary applications, e.g., electric vehicles, power grid storage, and load leveling, the issue of the limited natural abundance of lithium resource will arise in the immediate future, along with an inevitable cost increase [1]. Therefore, there arises a tremendous interest in alternative metal-ion batteries based on abundant resources. Sodium-ion battery (SIB) has recently attracted significant attention due to the "rocking chair" operating principle similar to the LIB. Moreover, virtually inexhaustible and ubiquitous sodium resources circumvent the geopolitical issue that plague lithium resource [2-5]. Because the chemistries of LIBs and SIBs are similar, the mechanistic insights obtained from the studies of LIBs may be applicable to the SIBs. In this regard, both SIBs and LIBs have shared some of the same cathode materials including layered $\mathrm{AMO}_{2}(\mathrm{M}=\mathrm{Co}, \mathrm{Ni}$, Ti $)$, olivine or maricite $\mathrm{AMPO}_{4}(\mathrm{M}=\mathrm{Fe}, \mathrm{Mn}, \mathrm{Co}, \mathrm{Ni})$, and the NASICON family of compounds [6,7].

In contrast to the great progress on SIB cathode materials, adapting LIB anode materials to SIBs has been thus far problematic. For example, a graphite anode with an interlayer distance of $1.86 \AA$ may not accommodate the reversible intercalation of $\mathrm{Na}^{+}$ions (diameter of $2.04 \AA$ ) [8]. As a result, pure graphite forms only a $\mathrm{NaC}_{64}$ phase during sodiation, thus resulting in a lower capacity, $\sim 35 \mathrm{mAh} \mathrm{g}^{-1}$, compared with $\sim 370 \mathrm{mAh} \mathrm{g}^{-1}$ in the Li-intercalated graphite compound of $\mathrm{LiC}_{6}$ (stage I). Graphene and hard carbon can circumvent the issue of size $[9,10]$, but their modest specific capacities are insufficient for practical SIB applications. Moreover, the well-studied metal oxide materials in LIBs with high theoretical capacities have also been investigated as viable anode materials for SIBs [11-14]. Due to similar, but not identical, chemistries in LIBs and SIBs, however, not all of them possess reliable electrochemical cycleability upon sodiation/desodiation. In this regard, in situ transmission electron microscopy (TEM) has been used to probe the failure mechanism of single-crystalline $\mathrm{SnO}_{2}$ nanowires for SIBs [15], despite its success in LIBs [16,17]. These reactions are difficult or impossible to be understood by ex situ measurements. Thus far, the effort directed toward in situ TEM research of SIBs is substantially less than that for LIBs, encouraging us to augment our knowledge of the various electrochemical reaction mechanisms stemming from the inherent characteristics of sodium.

In this paper, we employed state-of-the-art in situ TEM to visualize the microstructural evolution and phase transformation and to track ion diffusion during electrochemical sodiation of $\mathrm{ZnO}$ nanowires (ZNWs). A slow, step-by-step sodiation process was observed for single-crystalline ZNWs (sc-ZNWs). This process was characterized by a slowly propagating reaction front and the formation of heterogeneous interfaces due to non-uniform sodiation amorphization. In contrast, the sodiation process of poly-crystalline ZNWs (pc-ZNWs), composed of tiny ZnO nanograins, was also investigated, revealing an unexpected, fast sodiation process, thus enabling a better rate 
performance. Interconnected nanoscale channels among $\mathrm{ZnO}$ nanograins in pc-ZNWs are thought to act as unobstructed ionic transport pathways for fast sodium ion diffusion. To the best of our knowledge, this is the first in situ work in which otherwise similar electrode materials possibly respond differently during the electrochemical sodiation, depending on their single/poly-crystalline forms. This work provides breakthrough insights into crystallization forms-dependent ion diffusion kinetics and rate performance, which can be used to guide the design of high-performance SIB electrode materials through microstructural control.

\section{Experimental section}

\section{In Situ TEM Experiment of Electrochemical Sodiation/Desodiation}

The in situ electrochemical sodiation experiments were carried out using a Nanofactory TEM-scanning tunneling microscopy (STM) holder in an aberration-corrected JEOL ARM200CF TEM. In a typical experiment, the electrochemical setup consists of individual ZNWs as the working electrode, a layer of $\mathrm{Na}_{2} \mathrm{O}$ solid electrolyte, and bulk $\mathrm{Na}$ metal as the counter electrode. The ZNWs were glued to the half TEM copper grid with conductive epoxy as the working electrode. A $0.25 \mathrm{~mm}$-thick tungsten (W) wire was cut to produce a fresh tip that was then utilized inside a glove box filled with argon gas to scratch the Na metal surface to fetch fresh Na onto the tip. The layer of Na on the tip of the $\mathrm{W}$ wire was served as the counter electrode and sodium source. Both the ZNWs and sodium electrodes were mounted onto a Nanofactory STM-TEM holder inside the glove box. The holder was transferred into the TEM column within several seconds. A native $\mathrm{Na}_{2} \mathrm{O}$ layer formed on the surface of $\mathrm{Na}$ metal due to the exposure to air. This served as the solid-state electrolyte to allow transport of the $\mathrm{Na}^{+}$ions. The $\mathrm{Na}_{2} \mathrm{O} / \mathrm{Na}$ electrode was mounted on the mobile $\mathrm{STM}$ probe, which could be driven to contact the ZNW electrode inside the TEM. Electrochemical sodiation took place when a negative bias was applied to the ZNWs with respect to the sodium metal. This drove $\mathrm{Na}^{+}$ions transport through the solid-state $\mathrm{Na}_{2} \mathrm{O}$ layer. During the sodiation, electron energy loss spectroscopy (EELS) and electron diffraction (ED) were used to monitor chemical composition and phase transformation. Two advanced Gatan CCDs (Model 894 and Model OneView) were used for imaging and capturing the dynamic sodiation/desodiation process. Additional details are described elsewhere [18-20].

\section{Half Cell Measurement}

Galvanostatic electrochemical performance of the single- and poly-crystalline ZNW anodes was obtained with a 2025-type coin cell in the voltage window of $0.01-2.0 \mathrm{~V}$ versus $\mathrm{Na} / \mathrm{Na}^{+}$at room temperature on a Neware battery cycler at various current densities. The working electrode for the SIBs is the slurry mixture of active material ( $\mathrm{ZnO}$ powder), 
acetylene black, and polyvinylidene fluoride (PVDF) (75:15:10 weight ratio) in $N$-methyl pyrrolidine (NMP) which had been stirred for $12 \mathrm{~h}$. The slurry was coated onto the $\mathrm{Cu}$ current collector and dried at $240{ }^{\circ} \mathrm{C}$ for $4 \mathrm{~h}$ under Argon gas. The thickness of the electrode without current collector was $20 \pm 0.5 \mu \mathrm{m}$. Metal sodium foil was used as the counter electrode and reference electrode. The electrolyte consists of $1.0 \mathrm{~mol} / \mathrm{L} \mathrm{NaPF}_{4}$ in an ethylene carbonate (EC)-diethyl carbonate (DEC) mixture solution (1:1 volume ratio). For the galvanostatic intermittent titration technique (GITT) tests, the coin cells were discharged/charged at $\mathrm{C} / 10$ with a current pulse duration of $0.5 \mathrm{~h}$ and interval time of $1 \mathrm{~h}$.

\section{Results and discussion}

The first sodiation process is critical for the battery because this stage could imprint irreversible damage on the electrode materials due to microstructural evolution and phase transformation. To understand the electrochemical behavior of $\mathrm{ZnO}$ in SIBs, the sodiation process of individual sc-ZNWs was first observed in situ, as shown in Figure 1 and Movie S1. Figure 1a presents a schematic illustration of the in situ experiment setup that enables direct observation of the electrochemical sodiation of a nano-SIB. The taper-like sc-ZNWs are attached to the copper TEM grid and used as the working electrode. The Na metal attached to the tungsten wire serves as the counter-electrode and sodium source where the naturally oxidized $\mathrm{Na}_{2} \mathrm{O}$ and $\mathrm{NaOH}$ layer is used as a solid electrolyte. Figure $1 \mathrm{~b}$ shows a panoramic TEM image of the constructed nano-SIB inside the TEM. A magnified TEM image of the tip of the pristine ZNW is displayed in the inset of Figure 1b, showing its taper-like morphology with a taper degree of 1:3. The HRTEM image of the ZnO taper tip in Figure 1c shows its single-crystal nature with a well-resolved fringe spacing of $0.26 \mathrm{~nm}$, corresponding to the (0002) planes of $\mathrm{ZnO}$. The corresponding fast Fourier transform (FFT) pattern (inset in Figure 1c) can be indexed as the hexagonal structure of $\mathrm{ZnO}$ (JCPDF No. 79-0207).

After contact was established between the two electrodes, the electrochemical sodiation reaction driving the flow of electrons and $\mathrm{Na}^{+}$ions across the circuit was initiated by applying a bias of $-1.5 \mathrm{~V}$ to the sc-ZNW with respect to the $\mathrm{Na}$ electrode. The bias voltage was used for all sodiation experiments in this study unless otherwise stated. Figure 1d-j shows the morphological evolution of the sc-ZNW during the first sodiation. A nanoparticle (marked with a black arrow) on the nanowire is used as object of reference to measure axial elongation of the nanowire. At 0.5 min (Figure 1d), the sharp tip of the sc-ZNW has completely disappeared due to the sodiation-induced volume expansion. The sodiated region showed a lighter contrast with a length of around $290 \mathrm{~nm}$ (the reaction front is marked by a yellow arrow), yielding an average sodiation speed of $580 \mathrm{~nm} / \mathrm{min}$ along the longitudinal direction of the sc-ZNW. After an additional sodiation of $16 \mathrm{~min}$ (Figure 1i), the displacement of the reaction front was about $1230 \mathrm{~nm}$ and the sc-ZNW was elongated by $\sim 30.7 \%$. Unexpectedly, we find that the average sodiation speed varied during the sodiation process. The 
displacement of the reaction front as a function of sodiation time follows the rule of parabolic kinetics, as shown in Figure $2 \mathrm{a}$, indicating a diffusion-limited reaction process [21]. The average longitudinal sodiation speeds are displayed statistically in Figure 2b, revealing an intriguing step-by-step sodiation process (which will be addressed later).

The sodiation transformed the initially straight nanowire into a slightly bent shape with obvious radial expansion. The diameter located at the marked particle (Figure $1 \mathrm{j}$ ) was expanded by $\sim 37.5 \%$ after full sodiation, and the total volume expansion was estimated to be around $150 \%$. Interestingly, the sodiated region shows pseudo-moiré TEM contrast, a feather not observed in the unsodiated region. Further, the magnified TEM image in Figure 1j discloses the true features. Transverse dark stripes are embedded in the fully sodiated NW, which suggests a large local lattice strain. Note that a significant layer of $\mathrm{Na}_{2} \mathrm{O}$ formed on the surface of the nanowire (NW), which was partially associated with the fast diffusion of sodium along the NW surface. This phenomenon is due to the energetic nature of the in situ experimental probe and the imaging electron beam. Thus, the electron diffraction (ED) pattern of the fully sodiated NW was conducted on areas free of $\mathrm{Na}_{2} \mathrm{O}$ layer, as shown in Figure 1k. Diffraction rings in the ED pattern can be indexed as $\mathrm{NaZn}_{13}$ and $\mathrm{Na}_{2} \mathrm{O}$ phases. Thus, the electrochemical reaction in the sc-ZNW upon full sodiation can be expressed as equation (1):

$x \mathrm{ZnO}+(2 x+1) \mathrm{Na}^{+}+(2 x+1) \mathrm{e}^{-} \rightarrow x \mathrm{Na}_{2} \mathrm{O}+\mathrm{NaZn}_{x}$

This is a typical displacement reaction [22], leading to the formation of $\mathrm{NaZn}_{13}$ nanocrystals dispersed in the $\mathrm{Na}_{2} \mathrm{O}$ matrix. This is similar to the result of electrochemical sodiation of $\mathrm{SnO}_{2}$, another well-studied oxide NW [15]. Figure 11 schematically illustrates the crystal phase transformation of the $\mathrm{ZnO}$ anode during electrochemical sodiation process. Based on this electrochemical reaction, a high theoretical specific capacity of $\sim 685 \mathrm{mAh} \mathrm{g}^{-1}$ can be predicted for the fully sodiated ZNW ( $x=13)$, which significantly exceeds that of most SIB anodes presently available.

To better understand the stepwise electrochemical sodiation mechanism in sc-ZNWs, more in-depth localized observations were conducted to probe sodium ion transport pathways and microstructural evolution, as shown in Figure 3. After initial ZNW contact with the sodium electrode, the sodium species first diffused along the axial direction of the sc-ZNW. The $\mathrm{Na}_{2} \mathrm{O}$ layer formed gradually on the surface and wetted the ZNW, as shown in Figure 3a-c. Moreover, sodium diffusion also took place inside the sc-ZNW synchronously, which resulted in the longitudinal propagation of dense clouds of mobile dislocations, as observed in Movie S2. The dense clouds of mobile dislocations also occur in the reaction front of lithiated $\mathrm{SnO}_{2}$ nanowires [16]. The ED pattern changes of dislocation areas further verified the inward diffusion of sodium ions (Figure S1). Single-crystalline diffraction spots of the pristine sc-ZNW gradually evolved into 
diffraction spots superimposed on amorphous haloes, finally resulting in the formation of poly-crystalline diffraction rings after full sodiation. However, the dense clouds of dislocations were not observed during the lithiation of the ZNWs [23,24], indicating a different electrochemical behavior in the sodiation of the ZNWs. At 507 s (Figure 3c), the morphology of the ZNW still remained unchanged, except for a slight expansion in diameter. Obviously, the morphological evolution of the ZNW lagged behind sodium ion diffusion. Interestingly, the dynamic dislocation clouds gradually disappeared, leaving behind many transverse dark stripes dispersed inside the ZNW (Figure 3d). This could be because the sodium species initially diffusing along the surface of the ZNW had infiltrated inwardly, resulting in the transverse amorphization. When the longitudinal amorphization inside the ZNW was blocked by the transverse amorphization, the two amorphization regions caused heterogeneous interfaces (dark stripes in Figure 3d) due to their discontinuous atomic structures [25]. Electron energy loss spectroscopy (EELS) also corroborates the presence of sodium species prior to the violent electrochemical reaction (inset in Figure 3d). Up to this point, it took a long time for the ZNW to complete the slow wetting and infiltration processes that are considered as an incubation period for subsequent sodiation process.

After the incubation period, an ultrafast sodiation of the ZNW suddenly occurred and was completed only in $10 \mathrm{~s}$, as shown in Figure 3e-h and Movie S3. A diameter expansion of $28 \%$ exceeds the expansion of $11 \%$ observed during the whole incubation period of ZNW (nearly $10 \mathrm{~min}$ ). This resulted in discrete transverse cracks due to rapid inward diffusion of sodium along the transverse heterogeneous interfaces, as shown in Figure 3k,1. The stepwise electrochemical sodiation process can repeat itself and ultimately sodiate the entire ZNW (Figure 3i,j). The step-by-step sodiation process, including wetting, infiltration, and sodiation substeps, is schematically illustrated in Figure 4a. After physical aging of the sodiated regions, the heterogeneous interfaces still existed in the form of dark stripes inside the NW matrix (Figure 3k) and were embedded in light-colored areas whose components are a mixture of $\mathrm{Na}_{2} \mathrm{O}$ and $\mathrm{NaZn}_{13}$ phases (Figure 31). Moreover, the structural degradation caused by the heterogeneous interfaces could imprint irreversible damage on the electrode materials. Upon desodiation by applying an equivalent positive bias, we find that the extraction of sodium ions seems to be irreversible by observing the structural evolution and crystal phase change. Therefore, circumventing microstructure damage should be an important consideration for the enhancement of battery performance.

Figure 5 shows the microstructural evolution and phase transformation during the electrochemical sodiation process of pc-ZNWs prepared using our solution methods [26,27]. The pc-ZNWs are composed of tiny self-assembled ZnO nanograins, as shown in Figure 5a and Figure S2. The diffraction rings in the ED pattern of the pristine ZnO NW show its poly-crystalline nature (Figure $5 \mathrm{~g}$ ). When contact between the pc-ZNW and $\mathrm{Na}$ electrode was made, the entire 
pc-ZNW rapidly expanded uniformly within only several seconds, as shown in Figure 5b,c and Movie S4. After that, the ZNW morphology did not change (Figure 5d). The diameter expanded from $83 \mathrm{~nm}$ to $112 \mathrm{~nm}$, nearly $35 \%$ expansion rate. Judging by the ED pattern in Figure 5h, the fully sodiated products of the pc-ZNW are mixtures of $\mathrm{NaZn}_{13}$ and $\mathrm{Na}_{2} \mathrm{O}$ phases. Interestingly, the slow reaction front occurring in the sc-ZNW was not observed subsequently. This could be because that large numbers of interconnected channels among the $\mathrm{ZnO}$ nanograins provide abundant pathways for ion infiltration, thus alleviating the large mechanical strain along the longitudinal direction of the sodiated ZNW, resulting in an unexpectedly fast sodiation process. Experimental measurement and first-principles calculations have shown that nanograin boundaries have a lower ion diffusion energy barrier and can provide faster ion diffusion pathways $[28,29]$. Similarly, we suggest that sodium ion diffusion inside the pc-ZNW should benefit in like manner. In order to further probe the ion diffusion pathways among the nanograins, we have used an advanced CCD (Gatan, Model OneView) to capture the fast sodiation process (Movie S5). Abundant dark thin stripes distributed along grain boundaries after the sodiation, showing the ion diffusion pathways among $\mathrm{ZnO}$ grains (Figure S3). This kind of thin stripe is different from the transversely distributed thick stripes in the sc-ZNW (Figure 3d). Therefore, this observation of in situ sodiation of the pc-ZNW provides an important guide for designing high performance SIBs. The microstructure of the electrode materials should be optimized, such as by nanocrystallization [30].

Besides the vanished reaction front, we note the absence of the transverse dark stripes inside the fully sodiated pc-ZNW (Figure 5f). The dark stripes are the heterogeneous interfaces where the longitudinal sodiation amorphization inside the sc-ZNW is blocked by the transverse sodiation amorphization from rapid inward diffusion of sodium ions from the surface of the sc-ZNW. In contrast, the sodiation speed in the pc-ZNW is uniform due to the ultrafast Na-ion diffusion that benefits from the abundant ion transport pathways among $\mathrm{ZnO}$ nanograins with interconnected channels. In addition, we find no cracks on the surface of the sodiated pc-ZNW (Figure 5e), which strongly indicates that there is no dominant inward diffusion of sodium ions from the surface of pc-ZNWs. As a result, the discontinuous atomic structures between the different sodiation amorphization regions are not expected to occur inside the fully sodiated pc-ZNW. Moreover, we have tried to probe the effect of different grain sizes on the ionic diffusion and sodiation process. However, we do not distinguish the noticeable difference from the ZNW with bigger grains (Figure 5e-1, Figure S3 and Movie S5), except for the negligible change in sodiation speed. The ultrafast sodiation process of the pc-ZNWs is schematically illustrated in Figure 4b. All in all, the in situ TEM sodiation experiments can help us compare the difference between the sc-ZNWs and pc-ZNWs, especially in sodiation speed and microstructural evolution. The same electrode material responds differently to their single/poly-crystalline forms during the electrochemical sodiation. By the way, the channels among the $\mathrm{ZnO}$ nanograins can also partially buffer the 
sodiation-induced volume expansion [31,32]. Note that the reversible desodiation reaction is feasible for a sodiated pc-ZNW (Figure S4 and Movie S6), although the desodiation speed is slightly slower compared with the sodiation process, proving the viability of pc-ZNWs used for SIBs.

In principle, faster ion diffusion and sodiation processes result in improved rate performance in batteries. Thus, we further compared the rate capability of the two ZNW electrodes in SIBs. As shown in Figure 6a, at a low rate of C/10, the two ZNW electrodes exhibit a negligible difference in specific ion-extraction capacities. Nevertheless, at higher rates equal to and above $\mathrm{C} / 2$, the capacities of the sc-ZNW/Na cell are far lower than those of the pc-ZNW/Na cell. The sc-ZNW/Na cell displays 430, 370, and $290 \mathrm{mAh} \mathrm{g}^{-1}$ at C/2, 1C, and 2C, respectively, while the pc-ZNW/Na cell shows 475,440 , and $405 \mathrm{mAh} \mathrm{g}^{-1}$ at the same rates. Impressively, at $1 \mathrm{C}$ and $2 \mathrm{C}$, the pc-ZNW/Na cell retains $87 \%$ and $78 \%$ of its capacity at $1 \mathrm{C}$ and $2 \mathrm{C}$, respectively, while such retention ratios are only $68 \%$ and $57 \%$ for the sc-ZNW/Na cell (Figure 6b). To understand why the pc-ZNW cell exhibits a higher rate capability compared with the sc-ZNW cell, we employed GITT to measure the Na-ion diffusion coefficient in pc-ZNW and sc-ZNW cells. The ionic diffusion coefficient can be determined by solving Fick's second law with following equation (2) [33-37]:

$D_{\mathrm{Na}^{+}}=\frac{4}{\pi}\left(\frac{i V_{m}}{Z \mathrm{~F} S} \frac{\left(\frac{\mathrm{d} E}{\mathrm{~d} \delta}\right)}{\left(\frac{\mathrm{d} E}{\mathrm{~d} \sqrt{t}}\right)}\right)^{2}$ when $t \ll \frac{L^{2}}{D_{\mathrm{Na}^{+}}}$

where $L$ is finite diffusion length, $i$ is applied current, $V_{m}$ is molar volume of the material, F is Faraday's constant, $S$ is electrode surface area, $\mathrm{d} E / \mathrm{d} \delta$ is slope of the coulometric titration curve and $\mathrm{d} E / \mathrm{d} \sqrt{t}$ is the slope of the voltage versus square root of time plot during a constant current pulse. Figure $6 \mathrm{c}, \mathrm{d}$ shows the evolving diffusion coefficients as a function of potential, where similar trends are observed in the electrodes of pc-ZNWs and sc-ZNWs, indicating their similar sodium storage mechanism in $\mathrm{ZnO}$ material. However, higher diffusion coefficients ranging from $9.5 \times 10^{-14}$ to $0.5 \times 10^{-13} \mathrm{~cm}^{2} \mathrm{~s}^{-1}$ are obtained for the pc-ZNWs, compared with a range of $4.5 \times 10^{-16}$ to $0.5 \times 10^{-14} \mathrm{~cm}^{2} \mathrm{~s}^{-1}$ for the sc-ZNWs. The huge difference in diffusion coefficients is obviously related to the different crystallization forms in the ZNW. Returning to the in situ TEM sodiation experiments and focusing on the observed sodiation processes in a single ZNW, the Na-ion diffusivity can be roughly estimated using the Einstein-Smoluchowshi relation, $D_{\mathrm{Na}+}=L^{2} / 2 t$, where $L$ is the displacement of the reaction front and $t$ is the sodiation time. According to the in situ TEM observations, the sodiation reaction front of the sc-ZNWs yielded Na-ion diffusivities of $\sim 10^{-16} \mathrm{~cm}^{2} \mathrm{~s}^{-1}$, which are lower than values observed in the ex situ GITT measurements, suggesting that more complex processes than simple diffusion are 
occurring at the reaction front. Thus, additional kinetic studies, particularly involving multi-phase character calculation and simulation, will be required.

\section{Conclusion}

In summary, in situ TEM has been used to directly visualize the electrochemical sodiation process of ZNW anodes in nano-SIBs. We have compared the electrochemical sodiation behaviors of sc-ZNWs and pc-ZNWs. Upon sodiation, a reaction front propagates slowly along the sc-ZNWs, and heterogeneous interfaces are formed inside the ZNWs due to non-uniform sodiation amorphization. With $\mathrm{Na}$ insertion into $\mathrm{ZnO}$, a displacement reaction occurs, leading to the formation of crystalline $\mathrm{NaZn}_{13}$ nanograins dispersed in $\mathrm{Na}_{2} \mathrm{O}$ matrix. A step-by-step electrochemical sodiation behavior for the sc-ZNWs is revealed. Further, the electrochemical sodiation of pc-ZNWs composed of ZnO nanograins was also investigated using the in situ TEM, revealing an unexpectedly fast and uniform sodiation process, which is attributed to presence of abundant ion transport pathways among $\mathrm{ZnO}$ nanograins with interconnected channels. During the synthesis of the pc-ZNWs, interconnected nanoscale channels among $\mathrm{ZnO}$ nanograins were formed throughout the pc-ZNWs. These are expected to act as unobstructed transport pathways for ultrafast sodium ion diffusion. As a result, the reaction front and heterogeneous interfaces vanish. These findings demonstrate that chemically identical electrode materials can respond differently, depending on the single- or poly-crystalline form, during electrochemical sodiation, providing an important technique for designing high-performance SIB electrode materials, through microstructure control.

\section{Acknowledgement}

This work was supported by National Basic Research Program of China (973 Program, Grant No. 2015CB352106), National Natural Science Foundation of China (NSFC, Grant Nos. 61574034, 51372039), Jiangsu Province Science and Technology Support Program (Grant No. BK20141118). The work at Brookhaven National Laboratory is supported by U.S. DOE-BES under Contract number DE-AC02-98CH10886. Huolin Xin is supported by the Center for Functional Nanomaterials, which is a U.S. DOE Office of Science Facility, at Brookhaven National Laboratory under Contract No. DE-SC00127041. F. Xu also thanks Feng Wang and Wei Zhang from Brookhaven National Laboratory for their useful discussion and facility. 


\section{References}

1 J. M. Tarascon, Nat. Chem., 2010, 2, 510-510.

2 J. Sun, H. W. Lee, M. Pasta, H. Yuan, G. Y. Zheng, Y. M. Sun, Y. Z. Li and Y. Cui, Nat. Nanotech., 2015, 10, 980-985.

3 H. L. Pan, Y. S. Hu and and L. Q. Chen, Energy Environ. Sci., 2013, 2, 2338-2360.

4 S. W. Kim, D. H. Seo, X. H. Ma, G. Ceder and K. Kang, Adv. Energy. Mater., 2012, 2, 710-721.

5 Y. F. Li, Y. L. Liang, F. C. R. Hernandez, H. D. Yoo, Q. Y. An and Y. Yao, Nano Energy, 2015, 15, $453-461$.

6 S. P. Ong, V. L. Chevrier, G. Hautier, A. Jain, C. Moore, S. Kim, X. H. Ma and G. Ceder, Energy Environ. Sci., $2011,4,3680-3688$

7 V. L. Chevrier and G. Ceder, J. Electrochem. Soc., 2011, 158, A1011-A1014.

8 S. Komaba, W. Murata, T. Ishikawa, N. Yabuuchi, T. Ozeki, T. Nakayama, A. Ogata, K. Gotoh and K. Fujiwara, Adv. Funct. Mater., 2011, 21, 3859-3867.

9 J. T. Xu, M. Wang, N. P. Wickramaratne, M. Jaroniec, S. X. Dou and L. M. Dai, Adv. Mater., 2015, 27, $2042-2048$.

10 A. Ponrouvh, A. R. Goni and M. Rosa-Palacin, Electrochem. Commun., 2013, 27, 85-88.

11 Z. Li, J. Ding and D. Mitlin, Accounts Chem. Res., 2015, 48, 1657-1665.

12 J. C. Perez-Flores, C. Baehtz, A. Kuhn and F. Garcia-Alvarado, J. Mater. Chem. A, 2014, 2, 1825-1833.

13 M. M. Rahman, A. M. Glushenkov, T. Ramireddy and Y. Chen, Chem. Commun., 2014, 50, 5057-5060.

14 Z. L. Jian, B. Zhao, P. Liu, F. J. Li, M. B. Zheng, M. W. Chen, Y. Shi and H. S. Zhou, Chem. Commun., 2014, 50, $1215-1217$.

15 M. Gu, A. Kushima, Y. Shao, J. G. Zhang, J. Liu, N. D. Browning, J. Li and C. M. Wang, Nano Lett., 2013, 13, $5203-5211$

16 J. Y. Huang, L. Zhong, C. M. Wang, J. P. Sullivan, W. Xu, L. Q. Zhang, S. X. Mao, N. S. Hudak, X. H. Liu, A. Subramanian, H. Y. Fan, L. Qi, A.Kushima and J. Li, Science, 2010, 330, 1515-1520.

17 C. M. Wang, W. Xu, J. Liu, J. G. Zhang, L. V. Saraf, B. W. Arey, D. Choi, Z. G. Yang, J. Xiao, S. Thevuthasan and D. R. Baer, Nano Lett., 2011, 11, 1874-1880.

18 Q. M. Su, G. H. Du, J. Zhang, Y. J. Zhong, B. S. Xu, Y. H. Yang, S. Neupane and W. Z. Li, ACS Nano, 2014, 8, $3620-3627$.

19 W. W. Xia, F. Xu, C. Y. Zhu, H. L. Xin, Q. Y. Xu, P. P. Sun and L. T. Sun, Nano Energy, 2016, 27, 447-456.

20 F. Xu, B. H. Ge, J. Chen, A. Nathan, H. L. Xin, H. Y. Ma, H. H. Min, C. Y. Zhu, W. W. Xia, Z. R. Li, S. L. Li, K. H. Yu, L. J. Wu, Y. P. Cui, L. T. Sun and Y. M. Zhu, 2D Mater., 2016, 3, 025005. 
21 K. He, Y. N. Zhou, P. Gao, L. P. Wang, N. Pereira, G. G. Amatucci, K. W. Nam, X. Q. Yang, Y. M. Zhu, F. Wang and D. Su, ACS Nano, 2014, 8, 7251-7259.

22 M. C. Stan, R. Klopsch, A. Bhaskar, J. Li, S. Passerini and M. Winter, Adv. Energy Mater., 2013, 3, $231-238$.

23 A. Kushima, X. H. Liu, G. Zhu, Z. L. Wang, J. Y. Huang and J. Li, Nano Lett., 2011, 11, 4535-4541.

24 Q. M. Su, Z. M. Dong, J. Zhang, G. H. Du and B. S. Xu, Nanotechnology, 2013, 24, 255705.

25 K. Albe, Y. Ritter and D. Sopu, Mech. Mater., 2013, 67, 94-103.

26 F. Xu, Y. N. Lu, L. T. Sun and L. J. Zhi, Chem. Commun., 2010, 46, 3191-3193.

27 F. Xu, L. T. Sun, M. Dai and Y. N. Lu, J. Phys. Chem. C, 2010, 114, 15377-15382.

28 H. Paul and I. Sylvio, J. Phys: Condens. Mater., 2003, 15, R1257.

29 X. Zhu, C. S. Ong, X. Xu, B. Hu, J. Shang, H. Yang, S. Katlakunta, Y. Liu, X. Chen, L. Pan, J. Ding and R. W. Li, Sci. Rep., 2013, 3, 1084.

30 W. P. Kang, C. H. Zhao, R. Liu, F. F. Xu and Q. Shen, Crystengcomm, 2012, 14, 2245-2250.

31 H. J. Yang, S. C. Lim, S. Y. He and H. Y. Tuan, RSC Adv., 2015, 5, 33392-33399.

32 A. K. Mondal, D. W. Su, S. Q. Chen, X. Q. Xie and G. X. Wang, ACS Appl. Mater. Interf., 2014, 6, $14827-14835$.

33 W. Weppner, R. A. Huggins, J. Electrochem. Soc., 1977, 124, 1569-1578.

34 C. J. Wen, B. A. Boukamp, R. A. Huggins, W. Weppner, J. Electrochem. Soc., 1979, 126, 2258-2266.

35 Y. Q. Zhang, Q. Fu, Q. L. Xu, X. Yan, R. Y. Zhang, Z. D. Guo, F. Du, Y. J. Wei, D. Zhang and G. Chen, Nanoscale, 2015, 7, 12215-12224.

36 Z. L. Jian, Z. Y. Xing, C. Bommier, Z. F. Li and X. L. Ji, Adv. Energy Mater, 2016, 6, 1501874.

37 N. Böckenfeld and A. Balducci, J. Solid State Electrochem., 2014, 18, 959-964.

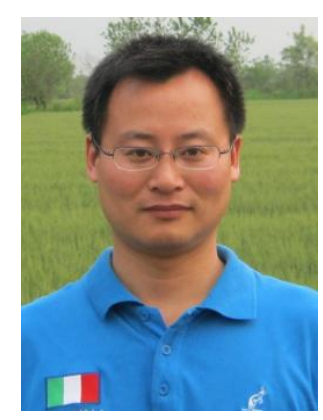

Dr. Feng Xu is an Associate Professor in the Key Laboratory of MEMS of the Ministry of Education at Southeast University (SEU). He obtained his Ph. D. from Nanjing Tech University and then was promoted to Associate Professor at Southeast University in 2012. He was a Visiting Scholar at Brookhaven National Laboratory from 2014 to 2015. Currently, his research interests cover structure-controlled preparation and in-situ transmission electron microscopy 
study of nanomaterials. In particular, his current interests center on application research of novel energy materials, including photoelectric conversion, energy storage, and environmental remediation, etc.

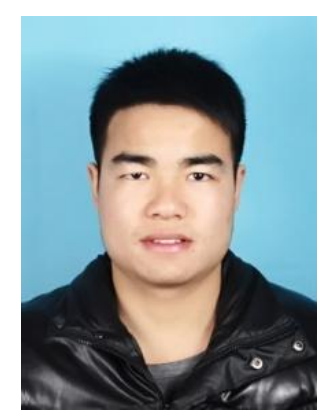

Zhengrui Li is currently pursuing his Master's degree in Key Laboratory of the Ministry of Education at Southeast University. His research interests focus on the synthesis of quantum dots of novel 2D materials and related biological applications.

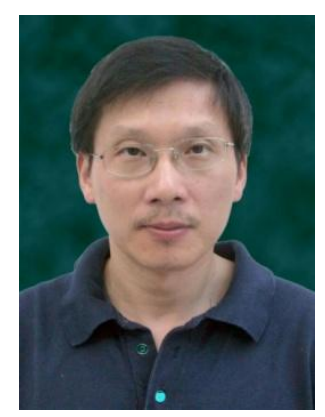

Dr. Lijun Wu is a Staff Scientist in Condensed Matter Physics and Materials Science Department at Brookhaven National Laboratory. He received his Ph. D. in 2002 from Hunan University, M.S. degree in 1987 and B.S. degree 1984 from Shanghai Jiaotong University. His research fields cover nanoscale materials science and engineering; electronic structure and superconductivity; electron-beam scattering and its interaction with matter; structural defects and interfaces in perovskites and transition-metal oxides; structural modeling and density functional theory calculations. His current interests focus on novel energy materials, including Li-ion batteries, fuel cells, photovoltaics, and thermoelectric materials, etc.

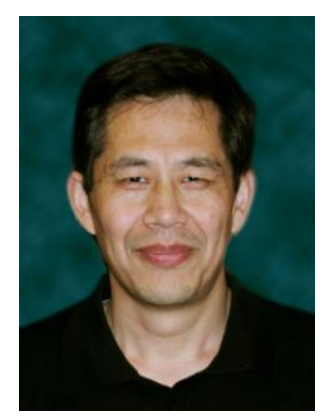


Dr. Qingping Meng is currently a visiting scientist in Brookhaven National Laboratory. He got his $\mathrm{Ph}$. $\mathrm{D}$. from Shanghai Jiao Tong University and then was promoted to Associate Professor at Shanghai Jiao Tong University in 2002. His research interests cover phase transformation in solids, thermal conductivity of thermoelectric materials, and Li-ion battery.

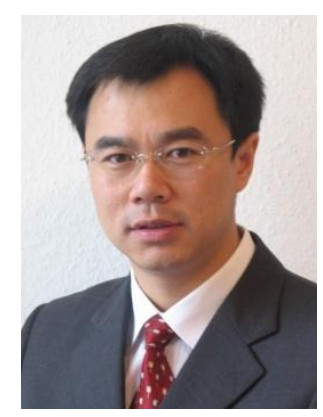

Dr. Litao Sun is currently a Distinguished Professor at Southeast University, China. He received his Ph. D. from the Shanghai Institute of Applied Physics, Chinese Academy of Sciences, followed by postdoctoral research at University of Mainz, Germany and a visiting professorship at the University of Strasbourg, France. His current research interests include in situ experimentation inside the electron microscope, graphene and related 2D materials, new phenomena from sub-10 nm nanoparticles, and applications of nanomaterials in environment, renewable energy and nano-electromechanical systems.

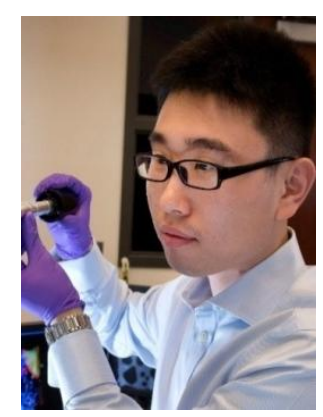

Dr. Huolin Xin is now a Staff Scientist in Brookhaven National Laboratory, US. He received his Ph. D. from Cornell University in 2011. He is interested in developing novel 3-D, atomic-resolution, in situ spectroscopic and imaging tools to probe the structural, chemical, and bonding changes of energy materials. His work on battery materials has been selected as the 2014's Top-10 Scientific Achievements by Brookhaven Lab. 


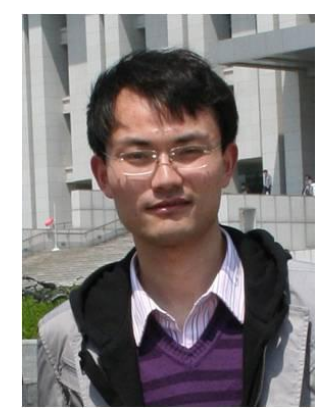

Jun Sun is currently a Ph. D. candidate in Key Laboratory of the Ministry of Education, Southeast University. He received his B.S. degree in Electronic Science and Engineering from Southeast University in 2008. He is interested in in situ TEM experiments, such as electron irradiation on nanomaterials, deformation mechanism of metal nanocrystal, current induced mass transportion, and related molecular simulation. In 2014 , as the first author, he has published a paper in Nature Materials.

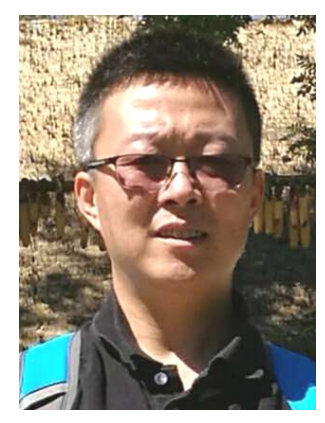

Dr. Binghui Ge is currently an Associate Professor in Institute of Physics, Chinese Academy of Sciences (IP, CAS). He obtained his Ph. D. from Institute of Physics in 2008 under the guidance of Academician Fanghua Li. After that, he continued his postdoctoral research in Tsinghua University, under the guidance of Academician Jing Zhu. From 2010, he returned to IP, CAS and joined Academician Fanghua Li's group. He is interested in the imaging theory of electron microscopy and electron crystallography, and studies of microstructure that controls materials' functionality.

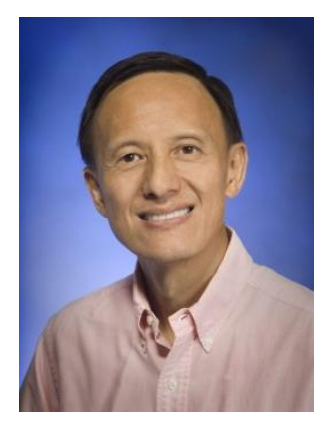

Dr. Yimei Zhu is a Senior Physicist in Condensed Matter Physics and Materials Science Department at Brookhaven National Laboratory, and an Adjunct Professor at Columbia University and at Stony Brook University in Stony Brook, New York. As one of the three Principal Investigators of the BNL's nanocenter proposal in 2000, he was considered as 
a co-founder of the Center for Functional Nanomaterials (CFN) at BNL. He acted as the Facility Leader and the group leader for the Electron Microscopy Facility at CFN. He is also an inaugural fellow of Microscopy Society of America and a fellow of the American Physical Society. His research focuses on studies of nanoscale phenomena that control materials' functionality by applying advanced electron microscopy and related techniques. 


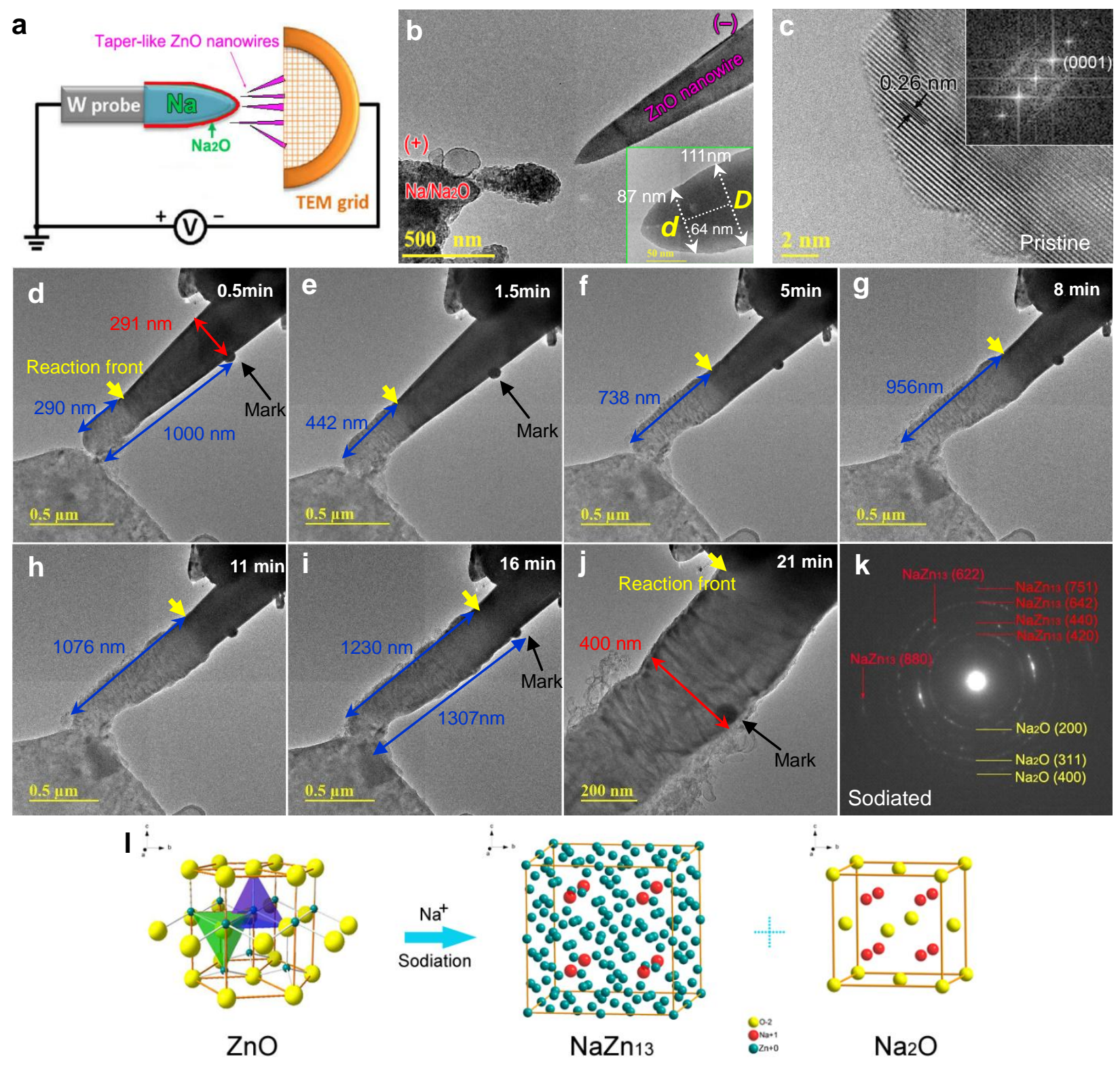

Figure 1 (a) Schematic illustration of the experimental setup for in situ electrochemical sodiation of nano-SIB. (b) Panoramic TEM image of the nano-SIB constructed with a taper-like ZNW inside a TEM. Inset in (b) shows taper-like morphology of the ZNW tip with a taper degree of 1:3. (c) HRTEM image of the ZNW tip shows its single-crystalline nature. Inset in (c) is the FFT pattern corresponding to the HRTEM image. (d-j) Time-resolved TEM images from video frames show morphology and structure evolution as a function of sodiation time. (k) Electron diffraction (ED) patterns of sodiated products demonstrate the formation of $\mathrm{NaZn}_{13}$ and $\mathrm{Na}_{2} \mathrm{O}$ phases. (1) Schematic illustration of the crystal phase transformation of the $\mathrm{ZnO}$ anode during electrochemical sodiation process. 

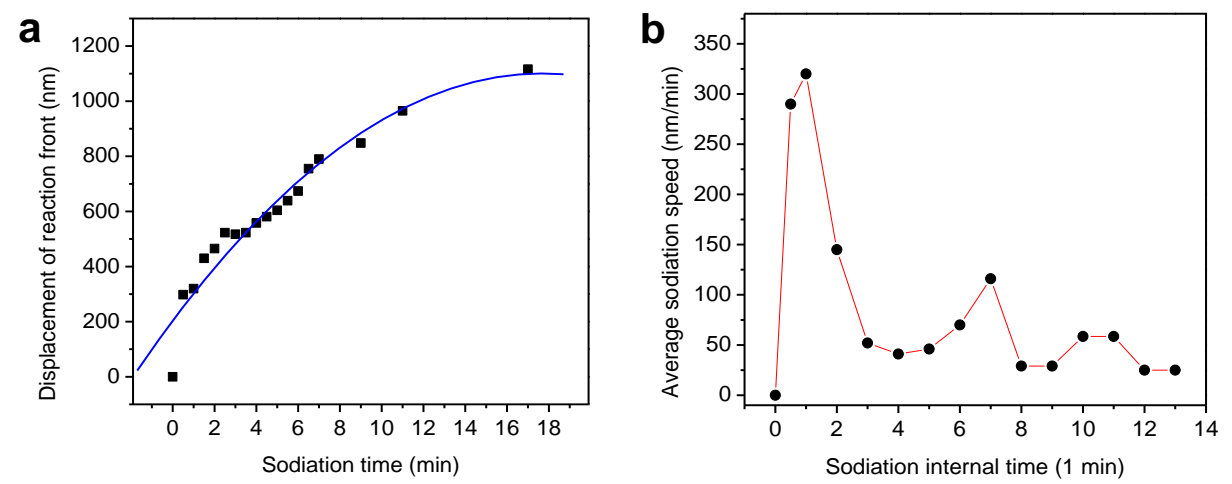

Figure 2 (a) Plot of the displacement of reaction front as a function of electrochemical sodiation time. (b) Average sodiation speeds at intervals of one minute. The measurements are along the longitudinal direction of the nanowire.

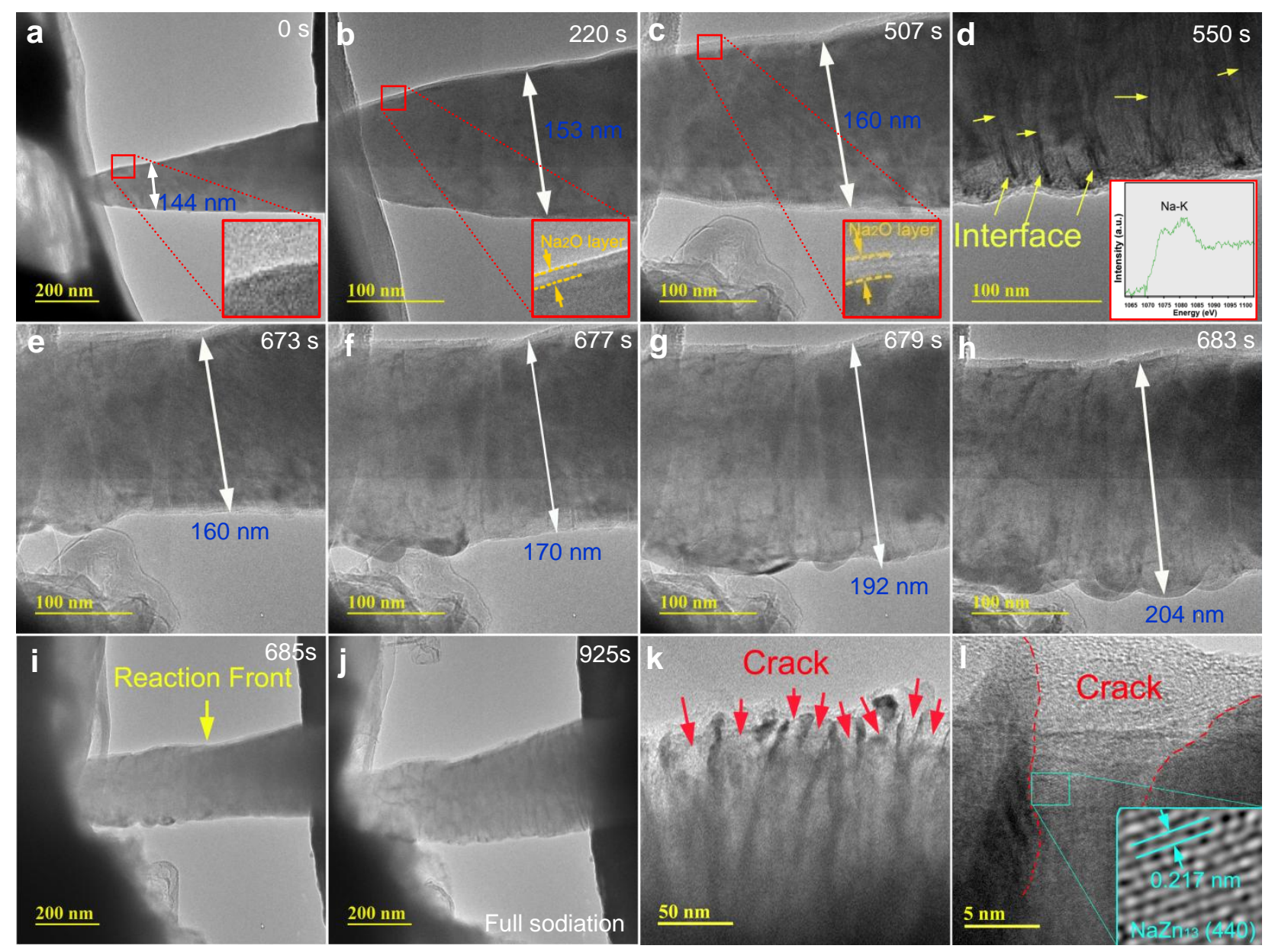

Figure 3 Time-resolved TEM images from video frames show a time-dependent step-by-step electrochemical sodiation process. $(a-c)$ Slow surface diffusion of sodium species first wetted the ZNW and (d) then infiltrated the ZNW from the surface. Inset shows electron energy loss spectroscopy of Na species. (e-h) After a slow wetting and infiltration process, fast sodiation occurs suddenly and is completed within only $10 \mathrm{~s}$, resulting in a huge diameter expansion. (i,j) Another stage including slow sodium diffusion, slow infiltration, and fast sodiation. (k,l) Discrete cracks due to rapid inward diffusion of sodium ions along the transverse heterogeneous interfaces. 
a
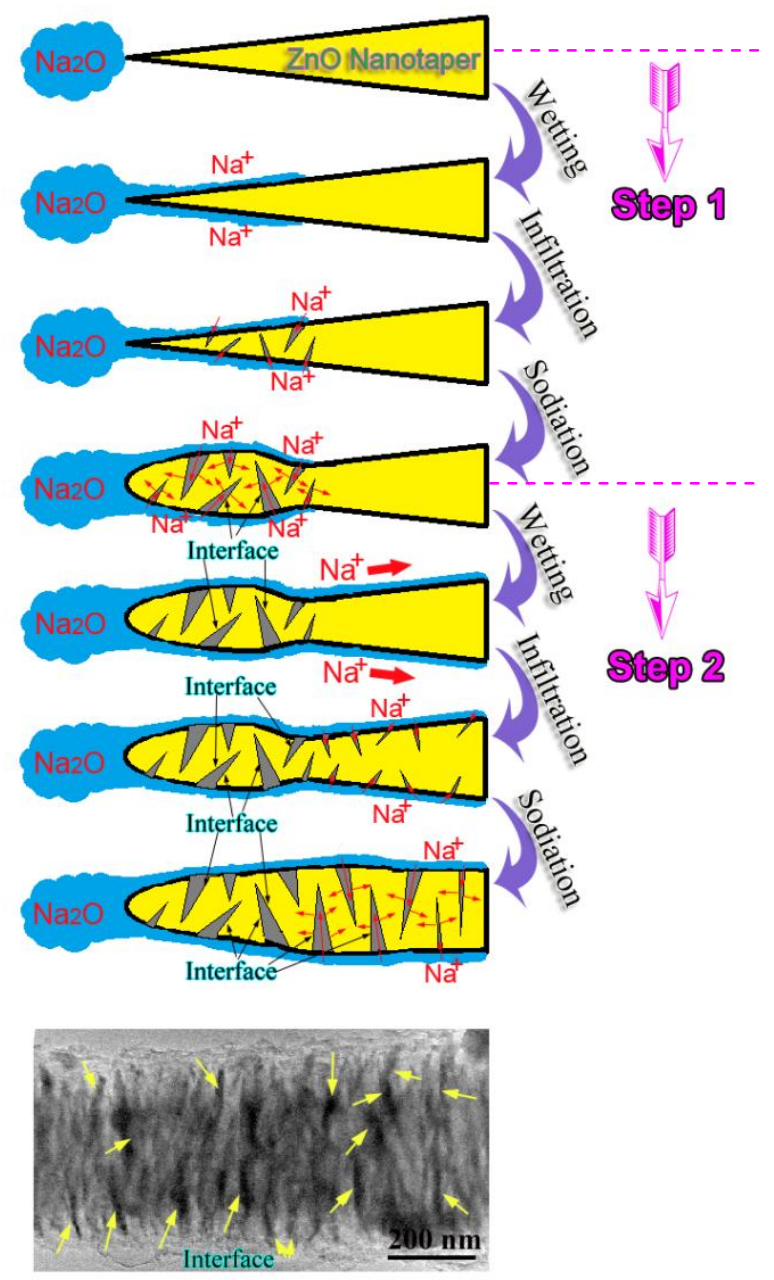

b

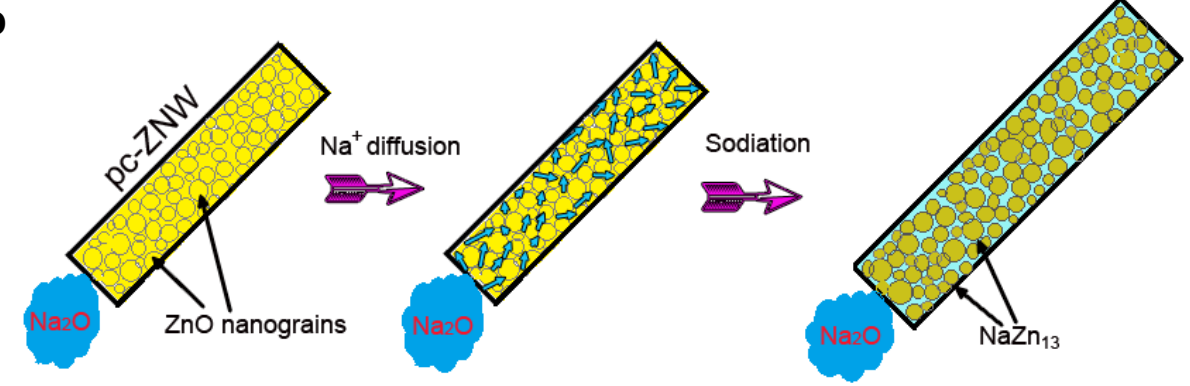

Figure 4 Schematic diagrams of (a) the longitudinal step-by-step sodiation process of an individual sc-ZNW and (b) the ultrafast and uniform sodiation process of an individual pc-ZNW. 


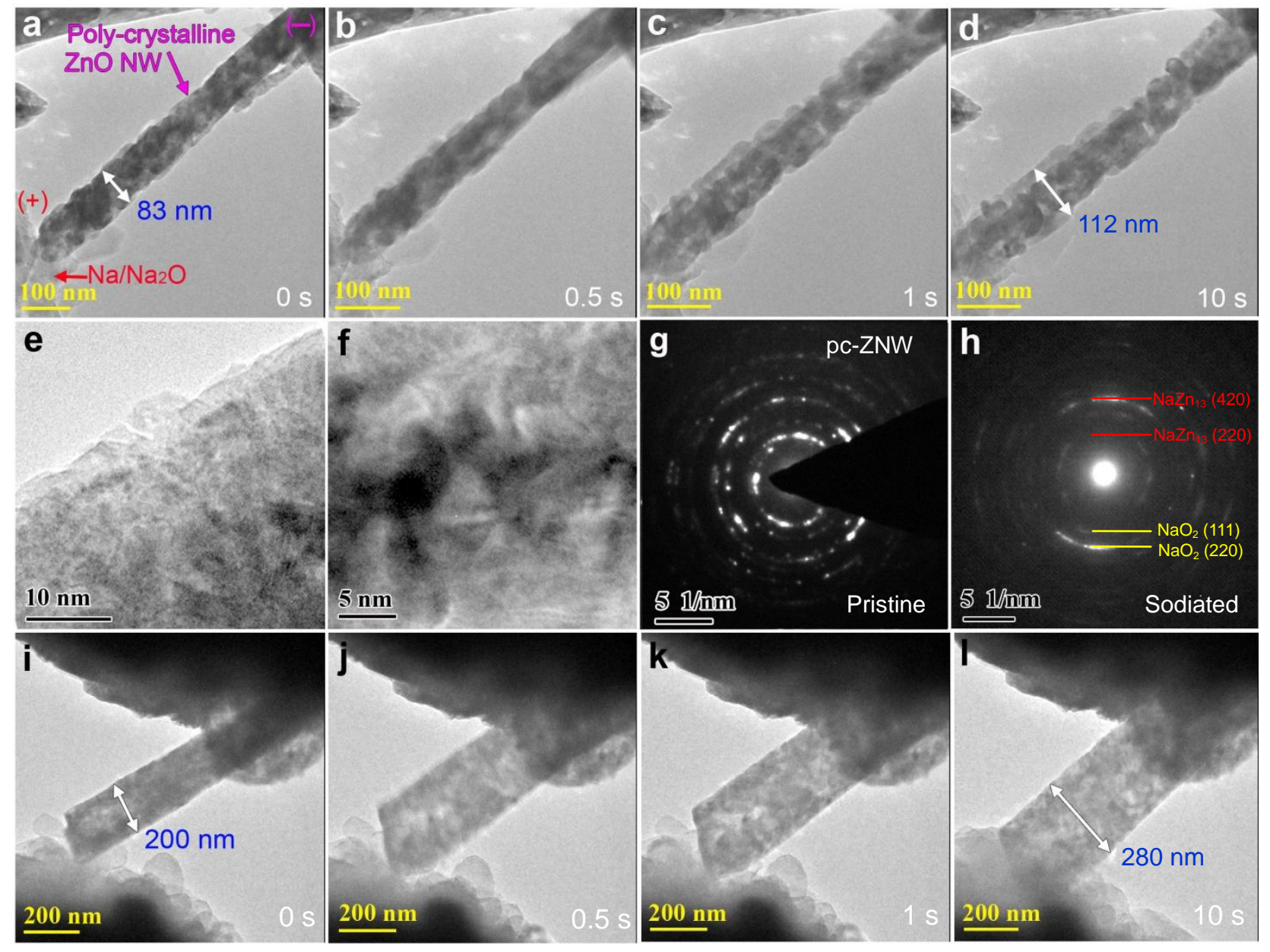

Figure 5 (a-d) Time-resolved TEM images from video frames show morphology and structure evolution of a poly-crystalline ZNW as a function of electrochemical sodiation time. A fast and uniform sodiation process of the entire NW was observed. (e) and (f) show local magnified TEM images. (g) and (h) are electron diffraction patterns before and after sodiation, respectively. (i-l) Time-resolved TEM images from video frames show morphology and structure evolution of another pc-ZNW. The ultrafast and uniform sodiation process also was observed. 

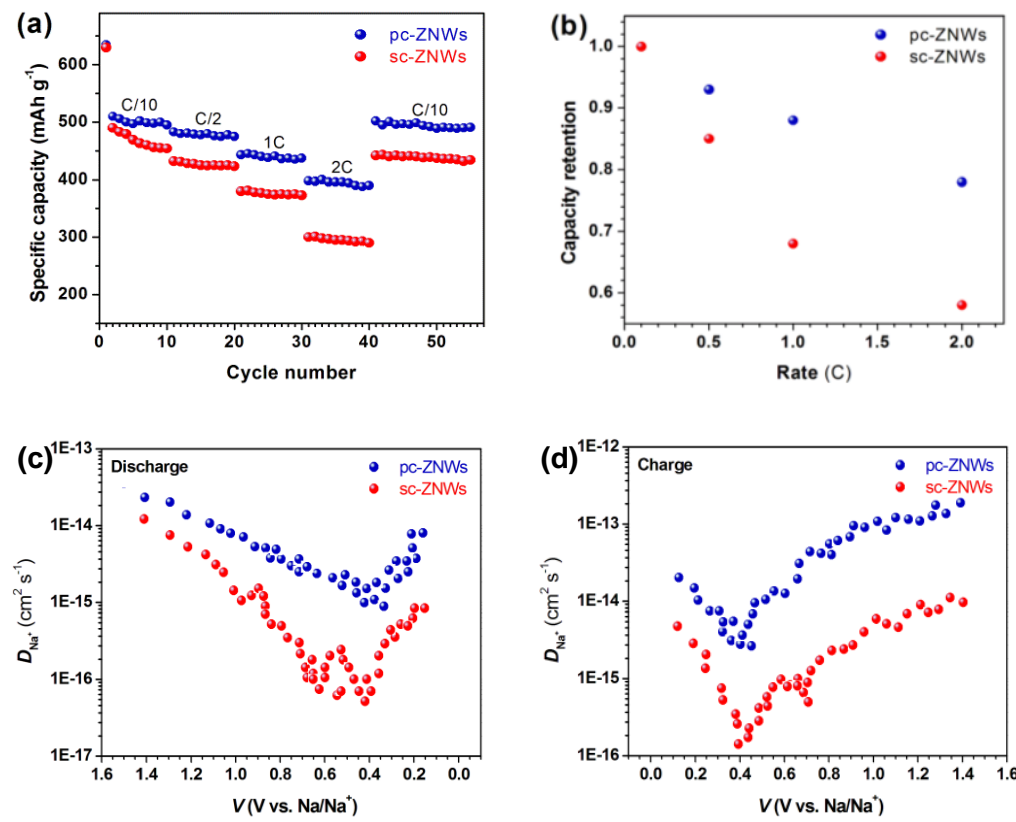

Figure 6 (a) Comparison of rate performance and (b) comparison of capacity retention between the sc-ZNWs and pc-ZNWs SIBs. (c, d) Comparison of diffusion coefficients calculated from the GITT potential profiles for the sc-ZNWs and pc-ZNWs SIBs as a function of state of potential. 


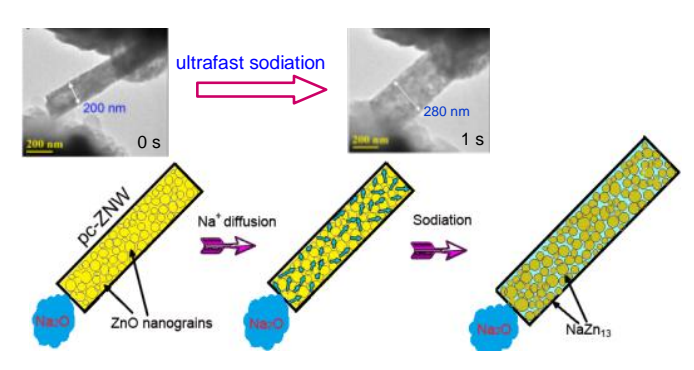

Ultrafast sodiation of pc-ZNWs benefiting from abundant ion transport pathways among $\mathrm{ZnO}$ grain boundaries. 